\title{
Ativação de Amidas - Um Instrumento Elegante para a Quimiosseletividade
}

Ana Mortinho Carlos Gonçalves*

\author{
Amide Activation: An Elegant Tool for Che- \\ moselectivity. The amide bond has always \\ been considered as a symbol of robustness, \\ especially due to the fact of being intimately \\ bound to the human skin. Its inherent stability \\ creates also new opportunities for further \\ exploitation. Furthermore, this bond can be \\ explored selectively in the presence of other \\ carbonyl containing groups. This paper \\ presents different ways of manipulation of \\ this type of bond, through various types of \\ activation (and subsequent reactivity).
}

\begin{abstract}
A ligação amida foi desde sempre sinónimo de robustez, especialmente devido ao facto de estar intimamente ligada à pele humana. A estabilidade que lhe é implícita, cria também novas oportunidades para o seu estudo. Para além do mais, esta ligação pode ser explorada seletivamente na presença de outros grupos funcionais contendo o grupo carbonilo. Este artigo apresenta diferentes formas de manipulação deste tipo de ligação, através do uso de diferentes modos de ativação (e consequente reatividade).
\end{abstract}

\section{Introdução}

Do ponto de vista sintético, poucas reações foram mais exploradas do que a alquilação de Friedel-Crafts, descoberta há mais de 100 anos. Um dos principais motivos reside no facto desta reação continuar a ser a base de um dos processos industriais mais importantes, utilizado especialmente na síntese de cetonas aromáticas [1]. Em 1877, dois químicos de nacionalidades diferentes, Charles Friedel (francês) e James Crafts (americano), publicaram um artigo que revolucionaria a forma de construir compostos aromáticos, originalmente partindo do benzeno - um precursor estável com origem na destilação de crude do petróleo [2]. Esta reação permitiu a formação de alquilbenzenos a partir de halogenetos de alquilo. Apesar da sua versatilidade, esta reação gerava produtos secundários indesejáveis, tais como rearranjos de carbocatião e produtos de polialquilação. Para evitar estas vias paralelas, a reação de acilação de Friedel-Crafts foi desenvolvida usando cloretos de acilo em conjugação com ácidos de Lewis (Esquema 1) [3]. Apesar de algumas desvantagens, ambas as transformações são bastante reconhecidas, razão pela qual estão presentes em virtualmente todos os livros de química orgânica.

Esquema 1 - Reação geral de acilação de Friedel-Crafts do benzeno.

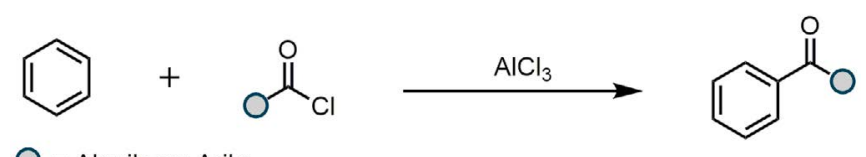

$\mathrm{O}=$ Alquilo ou Arilo

0 vasto trabalho de Friedel e Crafts estabeleceu esta reação como um método pioneiro para a formação de ligações carbono-carbono em anéis aromáticos [4]. A síntese de cetonas aromáticas continua, ainda hoje, a ser alvo de estudo, muito devido a problemas aliados à instabilidade dos cloretos de acilo e à toxicidade dos derivados do benzeno. Uma das possíveis alternativas encontra-se num grupo funcional aparentemente inócuo, a amida. 


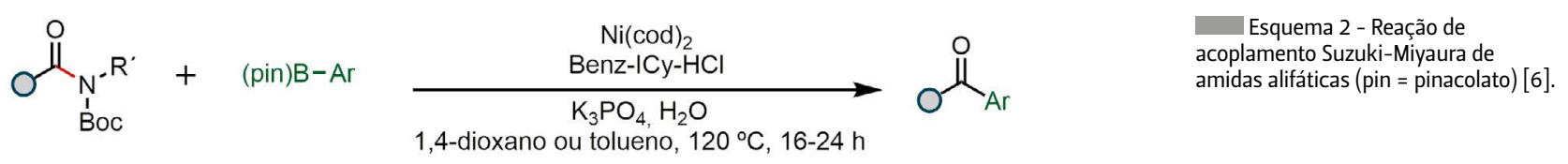

\section{Ativação de amidas usando metais}

Surpreendentemente, Garg e colaboradores apresentaram uma solução para este problema sintético, usando amidas como precursores de cetonas, levando à formação dos mesmos produtos oriundos da alquilação de Friedel-Crafts [5]. Para ter sucesso nesta árdua tarefa, foi usado um catalisador de níquel em conjugação com ésteres borónicos, resultando numa reação de acoplamento. Mais tarde, o mesmo grupo apresentou uma solução prática para a desvantagem da sensibilidade do catalisador $\mathrm{Ni}(\mathrm{cod})_{2}$ ( $\operatorname{cod}=1,5$-ciclooctadieno $)$ usando uma abordagem onde o catalisador é usado na forma de cápsulas de parafina (Esquema 2) [6]. Aplicando o catalisador estabilizado numa reação de acoplamento Suzuki-Miyaura, tendo como substrato amidas alifáticas, foi possível melhorar a versatilidade da reação [7]. 0 truque desta transformação encontra-se no material de partida, onde uma amida protegida com o grupo Boc (terc-butiloxicarbonilo) tem de ser usada, devido a esta ser "distorcida" (não planar). Para justificar este facto é necessário primeiro analisar a natureza da ligação amida.

0 grupo funcional amida possui uma geometria planar, com uma contribuição de cerca de $40 \%$ de ligaçãa dupla (Esquema 3). A conjugação $n_{N} \rightarrow \pi^{*}{ }_{C=0}$ concede à amida uma das ligações mais robustas e menos reativas em síntese orgânica. Paradoxalmente, a falta de reatividade deste grupo em relação a nucleófilos pode ser explorada através da adição de eletrófilos, um dos tópicos principais deste artigo.

O facto da estabilidade das amidas ser notória, paralelamente significa que reações seletivas que têm a clivagem da ligação amida como alvo permanecem extremamente desafiantes. De modo a introduzir uma desestabilização na ligação amida, foi descrita a utilização de amidas cíclicas ou de grupos protetores Boc ou Ts (tosilo).

Historicamente, a ideia de disrupção da ressonância da função amida, colocando a ligação amida num sistema cíclico com o átomo de nitrogénio no anel, remonta aos anos 1930-40. Ao longo dos anos, vários grupos de investigação interessaram-se pelo problema da tensão de anel nas amidas distorcidas, com contribuições de Pracejus, Yakhontov, Brown, Blackburn, Shea, Greenberg, Williams, entre outros [8]. Apenas no final do século XIX, Kirby reportou a síntese

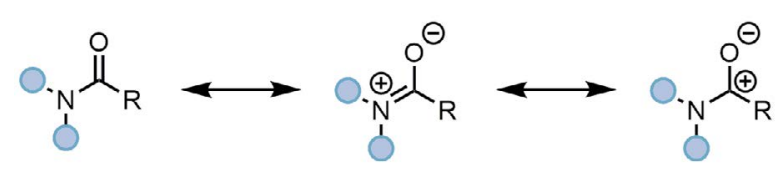

Esquema 3 - Ressonância da ligacção amida.
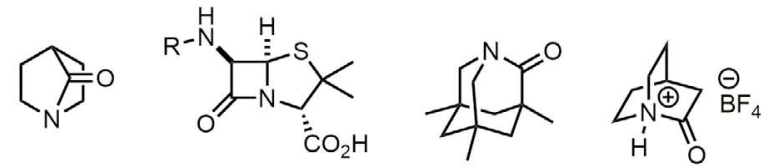

1938

Lukes

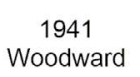
1998
Kirby

2006 Stoltz

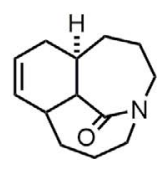

2005
Figura 1 - Exemplos clássicos de amidas distorcidas [9-13].

\section{Amida}<smiles>CC(=O)N([O-])C(=O)O</smiles>

Desestabilização<smiles>CC(=O)N([O-])O</smiles>

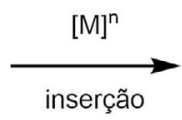<smiles>CC(=O)[Al](=[Te])N([O])O</smiles>

Esquema 4 - Mecanismo geral de ativação de amidas por torção nas reações de acoplamento cruzado [14,15].

e a estrutura da 1-aza-2-adamantanona que continha uma ligação amida perfeitamente perpendicular [9]. Como esperado, a amida exibia características de reatividade de uma "aminocetona". Em 2006, o grupo de Stoltz procedeu à síntese de uma amida "distorcida" usada como referência, o tetrafluoroborato de 2-quinuclidónio (Figura 1) [10]. Estes dois exemplos de amidas foram usados para testar os limites de quebra da ressonância da ligação amida e são agora considerados clássicos da química orgânica.

Recentemente surgiu um interesse na ativação da ligação N-C(O) das amidas através da inserção seletiva de um metal na ligação N-C(0) (Esquema 4) [14,15]. 0 uso da torção na ligação amida permitiu o avanço da química orgânica em geral, sendo agora uma ligaç̧ão C-N atrativa e com possibilidade de ser explorada em reações de acoplamento cruzado. 
Estudos recentes demonstraram a possibilidade de reações do tipo Suzuki, Heck, Suzuki descarboxilativo, Negishi e ativação C-H direta através da clivagem C-N da ligáção amida por inserção de metal em amidas estereamente (e/ou eletronicamente) distorcidas. Apesar de várias amidas apresentarem uma disrupção na ressonância e poderem participar neste tipo de reação catalisada com metais de transição, os melhores resultados foram obtidos com N-g'lutarimidas (Figura 2) [16].

Estas reações de ativação da ligaçã̃o C-N de amidas têm recebido uma especial atenção devido à sua elevada importância do ponto de vista químico (incluindo na descoberta de fármacos, na agroquímica, na bioquímica, na química estrutural e na ciência de polímeros) [17].

Os métodos aqui considerados demonstram que a liğação amida, tipicamente inerte, pode ser ativada seletivamente e clivada através do uso de catálise por metais de transição. Estas poderosas metodologias complementares dão acesso à desconexão da ligação amida para a formação de novas ligacções C-C ou C-N através de acoplamentos não convencionais.

Em 2016, Garg e colaboradores desenvolveram um procedimento que recorria a uma reação de transamidação de carboxamidas secundárias utilizando um catalisador de níquel [18]. Até então, poucos exemplos de transamidação seletiva de carboxamidas tinham sido reportados (Esquema 5).
Este sistema catalítico foi, aliás, o primeiro método generalizado para o acesso a amidas funcionalizadas usando carboxamidas secundárias protegidas com o grupo Boc. É possível encontrar semelhanças com a catálise efetuada por outros metais, onde um intermediário acil-níquel é então convertido em amidas na presença da amina apropriada. Esta metodologia é bastante abrangente em termos de substratos, incluindo heteroátomos básicos, aminas heterocíclicas e nucleófilos derivados de aminoácidos. Apesar de tudo, o uso do catalisador $\mathrm{Ni}(\mathrm{cod})_{2}$ limita o procedimento à utilização de caixa-de-luvas [18].

Mais tarde, o grupo de Garğ reportou uma transamidação de amidas alifáticas com aminas na presença de $\mathrm{Ni}-\mathrm{NHC}(\mathrm{NHC}=$ carbeno $\mathrm{N}$-heterocíclico) [19]. Neste método, utilizaram Ni(cod) 2 como catalisador e Benz-ICy.HCl como ligando. 0 mesmo grupo decidiu ainda estender as reações de acoplamento C-C, C-N e C-O (esterificação, transamidação e Negishi) tirando partido da vantagem do encapsulamento do catalisador/ligando. Para tal, utilizaram o ligando SIPr (1,3-bis[2,6-bis(1-metiletil) fenil]-2-imidazolidinilideno), sendo este também instável fora de uma caixa-de-luvas [19].

Em 2017 foi desenvolvida uma metodologia para a síntese de $\alpha$-aminonitrilos [20]. Esta estratégia, estabelecida por Dixon e colaboradores, submete amidas terciárias a uma reação de Strecker catalisada por um<smiles>O=C(O)N1C(=O)CCCC1=O</smiles><smiles>O=C(O)N1C(=O)CCC1=O</smiles><smiles>O=C(O)N1C(=O)c2ccccc2C1=O</smiles>

$\mathrm{O}=\mathrm{Me}$, Et, ciclo-hexilo, fenilo, terc-Bu

Figura 2 - Amidas distorcidas e exemplos de $\mathrm{N}$-glutarimidas utilizadas em reações catalisadas por metais de transição [16].

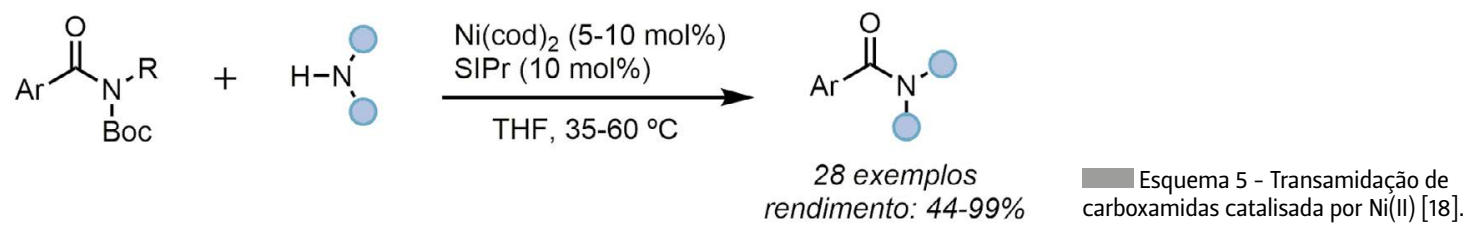

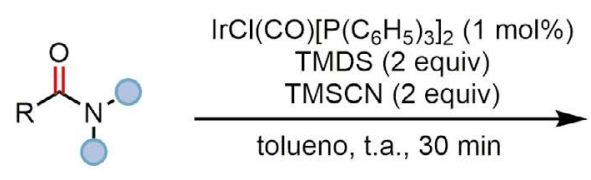

1

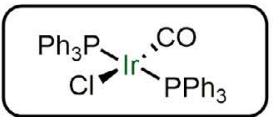

complexo de Vaska

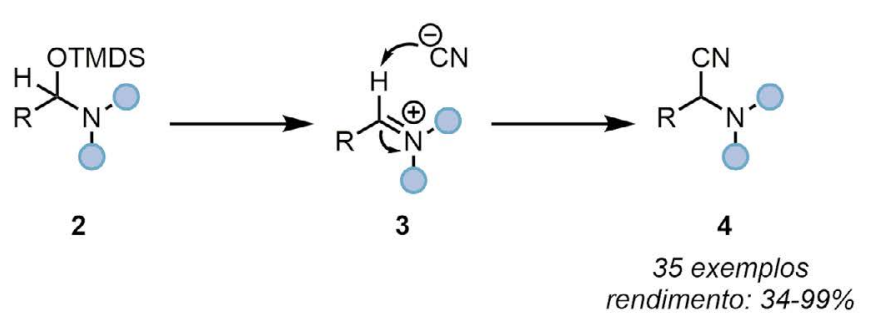

Esquema 6 - Redução de amidas terciárias na presença do complexo de Vaska [20]. 
complexo de irídio (IrCl(CO) $\left.\left[\mathrm{P}\left(\mathrm{C}_{6} \mathrm{H}_{5}\right)_{3}\right]_{2}\right)$, mais correntemente denominado complexo de Vaska (Esquema 6). A reação baseia-se na redução parcial da ligáção amida (1) na presença de tetrametildisiloxano (TMDS), gerando o hemiaminal (2), que é posteriormente substituído por cianeto após tratamento com TMSCN (TMS = trimetilsililo) gerando o composto (4). Este procedimento foi facilmente adaptável a amidas, lactamas, alcaloides e péptidos. Desde então, outros métodos da redução da amida a grupo nitrilo emergiram, assim como estratégias de conversão de amidas terciárias a enaminas e iões imínio usando o complexo de Vaska [21].

No mesmo ano (2017), o grupo de Garg criou uma estratégia de redução de amidas secundárias e terciárias a aminas na presença de fenilsilano e quantidades catalíticas de $\mathrm{NiCl}_{2}(\mathrm{dme})$ (dme = dimetoxietano) [22]. Esta estratégia permitiu também reduzir com sucesso lactamas com diferentes tamanhos de anel (Esquema 7), conduzindo a um aumento na variedade de reações de amidas catalisadas por níquel.

Apesar da importância das reações de acoplamento ser reconhecida, métodos alternativos para a adição de vários nucleófilos a derivados de ácido carboxílico permanecem inexplorados. Foi então considerado que as amidas poderiam ser um substrato viável para ultrapassar este obstáculo. Como tal, Garg e colaboradores descreveram pela primeira vez um método para a adição direta intermolecular de dois nucleófilos diferentes ao grupo carbonilo da amida (Esquema 8) [23]. Esta transformação permite a fácil conversão de amidas em álcoois quirais via reação de cascata Suzuki-Miyaura e subsequente hidrogenação. A reação demonstrou ser tolerante a estereocentros epimerizáveis e a diferentes grupos funcionais. Como alternativa, foi ainda possível a síntese enantiosseletiva de álcoois recorrendo a um método quimioenzimático [24].

Os métodos de ativação de amidas catalisadas por $\mathrm{Ni}$ e Pd devem ser vistos como complementares pois o níquel é mais económico e sustentável, mas as reações catalisadas por paládio garantem geralmente uma maior extensão reacional. Em 2016, o grupo de Szostak reportou uma reação de Heck catalisada por paládio via ativação C-N. Esta reação representava a primeira metodologia para o acoplamento descarboxilativo de amidas [25]. Utilizou-se para tal um método sem base ou ligando na presença de $\mathrm{PdCl}_{2} / \mathrm{LiBr}$ (Esquema 9a).

A metodología anterior foi aplicada à cianação de amidas para a obtenção de nitrilos aromáticos (Esquema 9b) [26]. Neste procedimento, foi utilizado cianeto de zinco como reagente de cianação que, adicionado a amidas, permitiu aceder a reações de acoplamento. Esta transformação demonstrou ser bastante tolerante a vários grrupos funcionais incluindo a síntese de diversos compostos arilo, heteroarilo e vinil nitrilos. É ainda
Esquema 7 - Redução de amidas secundárias e terciárias a aminas catalisadas por $\mathrm{Ni}(\mathrm{II})$ [22]
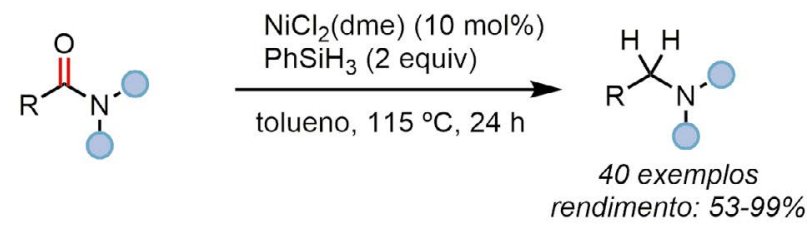

Esquema 8 - Reação de acoplamento Suzuki-Miyaura em cascata de amidas com adição sequencial de nucleófilos (Nu) [23]

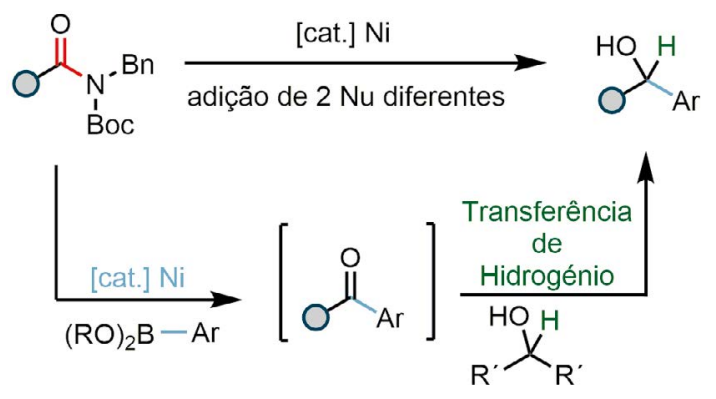

$\mathrm{O}=$ Alquilo ou Heteroaromático

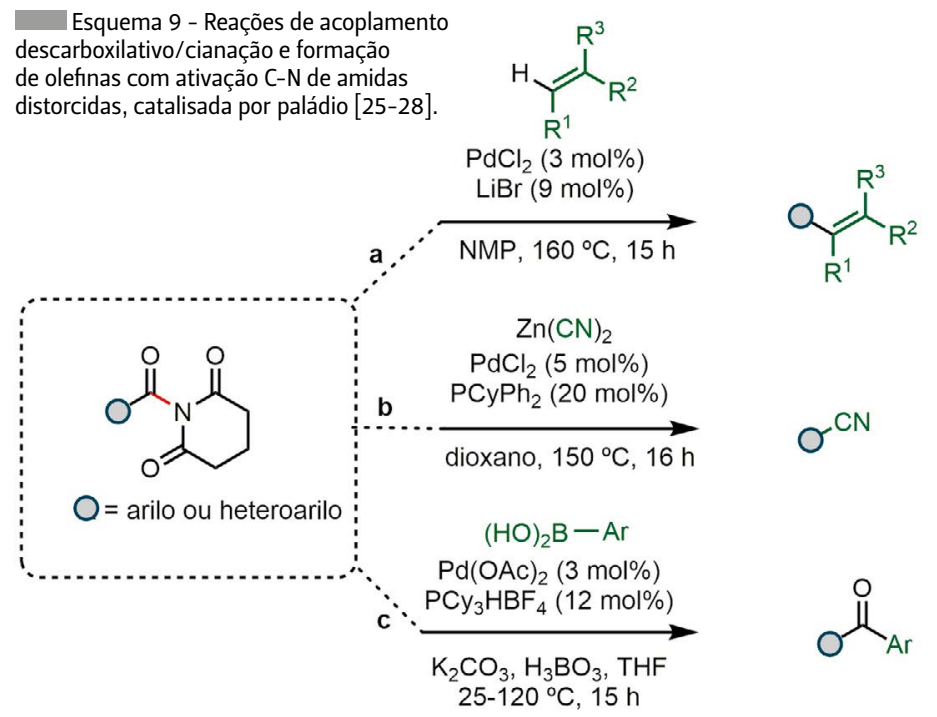

possível submeter as mesmas amidas a uma reação de fosforilação [27] gerando os respetivos fosfatos de arilo. Durante os estudos desenvolvidos na síntese de cetonas catalisada por paládio, estabeleceu-se que o uso de ácidos borónicos como parceiro reacional era crucial para a reação. No mesmo estudo foram ainda realizadas várias experiências de modo a esclarecer o acoplamento de amidas catalisado por paládio (Esquema 9c) [28]. 


\section{Ativação de amidas na ausência de metais}

Uma outra alternativa à reação de Friedel-Crafts é a adição direta de reagentes organometálicos a derivados do ácido carboxílico, representando um dos métodos mais utilizados para a síntese de cetonas. Neste sentido, Szostak e seus colaboradores reportaram um método quimiosseletivo para a síntese de cetonas através da adição direta de compostos organometálicos a $\mathrm{N}$-acil-azetidinas (Esquema 10) [29].

0 mesmo grupo reportou uma reação de transamidação de amidas secundárias recorrendo uma vez mais a amidas protegidas com os grupos Boc ou Ts, na presença de trietilamina [30] e da base LiHMDS (bis(trimetilsilil)amida de lítio) [31].

Utilizando o mesmo raciocínio para a ativação de amidas na presença de uma base e através da clivagem da lig̉ação C-N, o grupo de Szostak desenvolveu um método de transformação direta de amidas a ésteres aromáticos na presença de $\mathrm{K}_{3} \mathrm{PO}_{4}$ [32].

Recentemente, Dixon e colaboradores desenvolveram um método de ativação de amidas para gerar um intermediário reativo que pudesse formar lighações C-C [33]. Para esse propósito utilizaram amidas aromáticas em condições redutivas ( $\mathrm{NaH} / \mathrm{Nal}$ ). Esta reação leva à formação um intermediário hemiaminal aniónico que reage seguidamente com TMSCl e é posteriormente intercetado com reagentes de Grignard ou cianeto de tetrabutilamónio (Esquema 11).

\section{Ativação usando agentes eletrofílicos}

De forma paralela, as amidas podem ser ativadas por eletrófilos fortes sem a necessidade de catálise metálica, sendo este método compatível com o ca- rácter nucleofílico inato das amidas. Olhando para o preâmbulo deste tipo de reações, pode afirmar-se que grande parte da reatividade deste grupo funcional foi explorada recorrendo a estes mesmos eletrófilos. Devido ao caráter instável de alguns catalisadores previamente referidos, aliado à escassez dos mesmos no curso de grande parte do século XX, recorreu-se à utilização de compostos orgânicos para manipular a ligação amida. Um dos primeiros exemplos data de 1893, onde se demonstrou que se poderia obter um intermediário bastante reativo utilizando $\mathrm{P}_{2} \mathrm{O}_{5}$, um agente desidratante, em combinação com uma amida [34]. Esta tornou-se uma das reações mais bem conhecidas da química orgânica (Bischler-Napieralsky), que ainda hoje encontra uso em processos químicos e em laboratórios de síntese em todo o mundo.

A natureza da ligação amida é de grande importância. 0 uso de amidas primárias, secundárias e terciárias origina produtos completamente distintos, muitos deles foram erroneamente caracterizados no passado devido a métodos analíticos insuficientes [35]. De entre os diferentes tipos de amidas, as terciárias foram alvo de maior escrutínio, dado que a sua ativação envolve uma panóplia de diferentes espécies com um elevado interesse sintético. Um dos primeiros mecanismos plausíveis para a formação de $\alpha$-cloro-enaminas foi proposto por Viehe [36]. Este recorria a fosgénio e trietilamina como base. Nesta proposta, a amida era inicialmente transformada num cloreto de imínio que, após subsequente desprotonação pela trietilamina, gerava a enamina clorada (Esquema 12). Este último intermediário estaria em constante equilíbrio com o ião cetenimínio, sendo este crucial do ponto de vista da reatividade.

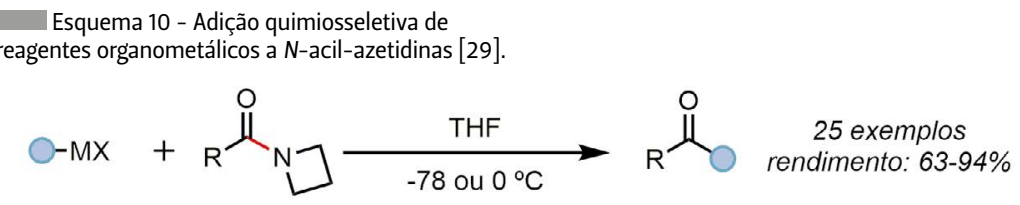

$M=M g$ ou Li
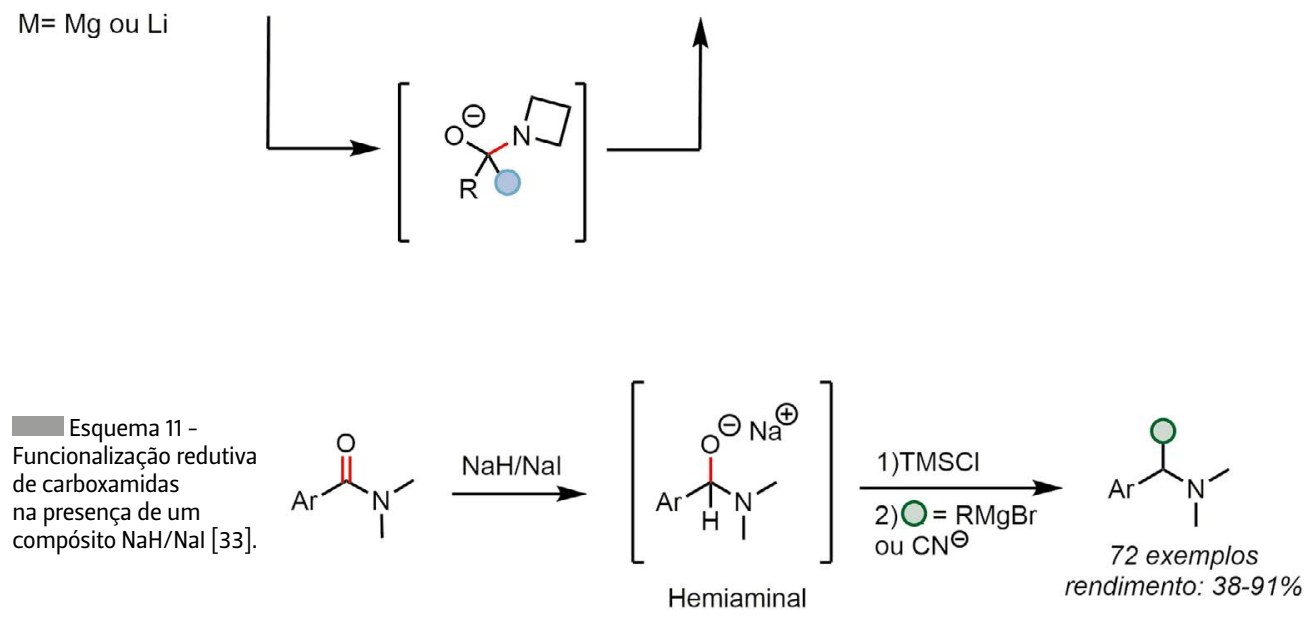

QuímICA | Vol. 45 | N.161 | 2021 | 113 


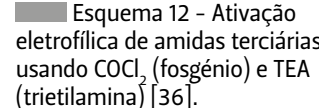
(trietilamina) [36].

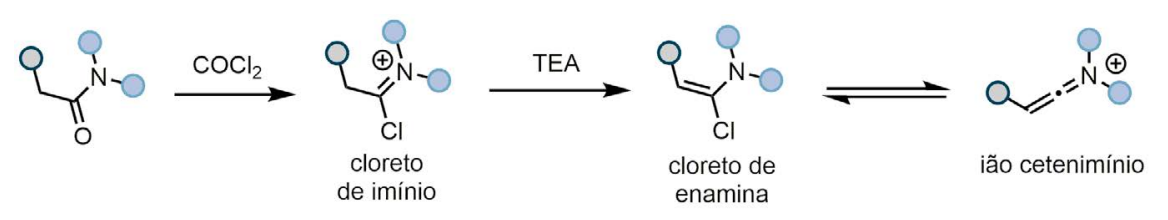

Esquema 13 - Inibição da reação secundária "aldólica" ao usar anidrido tríflico $\left(\mathrm{Tf}_{2} \mathrm{O}\right)$ [38].
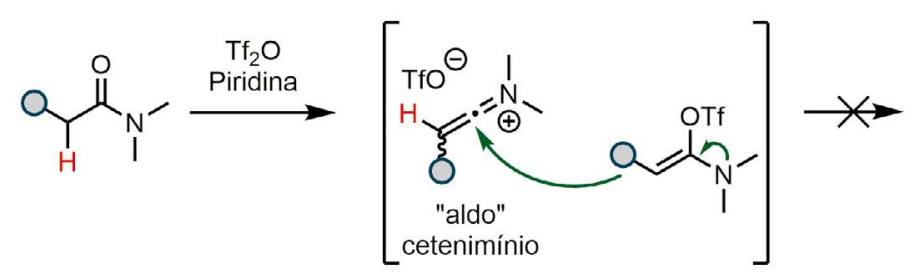

Não observada

Menos nucleofílico

Esquema 14 - Cicloadição
intramolecular [2+2] após

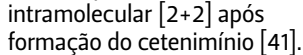
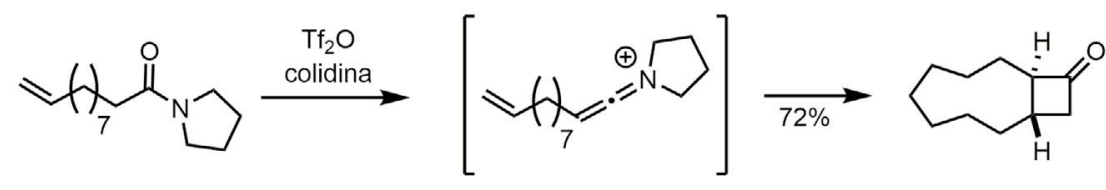

Não acessível partindo do ceteno [2+2]

15 exemplos
Uma das desvantagens da ativação assenta na reação secundária do intermediário cetenimínio e da enamina clorada, que são gerados a partir do mesmo material de partida. Devido ao caráter nucleofílico e eletrofílico das duas espécies, era muitas vezes observada a formação do produto "aldólico", levando a rendimentos inferiores ao esperado [37]. A solução, que ainda é usada nos dias de hoje, é a utilização de um ativador eletrofílico diferente - o anidrido tríflico ou anidrido trifluorometanossulfónico. Um fator não mencionado até este momento é o contra-ião - que existe ubiquamente em solução. Ao modular o caráter do contra-ião, é possível inibir a nucleofilicidade da enamina formada, evitando consequentemente a reação adversa (Esquema 13). O pioneiro na utilização deste ião foi Ghosez, conseguindo, com grande sucesso, o aumento do rendimento da reação [37]. Este facto levou a que até aos dias de hoje condições semelhantes sejam usadas neste tipo de transformação.

Olhando para as condições reacionais, outro fator que tem influência é a base utilizada no processo. Dentro do grande leque de bases orgânicas, um subgrupo de bastante sucesso neste tipo de transformação foram as piridinas [39]. Olhando mais uma vez para o mecanismo, pode observar-se que o equilíbrio de formação do cetenimínio é de grande importância, pois a base tem de possuir a capacidade de estabilizar o intermediário. As piridinas têm essa capacidade pois a ligação que estabelecem com o cetenimínio é bastante favorável devido ao carácter iónico da ligação. Posto isto, é relevante mencionar que a piridina não substituída leva, geralmente, a adutos demasiado estáveis, fazendo com que as reações subsequentes não ocorram [40]. Esta característica mais específica faz com que se recorra a piridinas substituídas nas posições 2, 4 e 6. Esta capacidade de modular a reatividade com a alteração da piridina é de extrema importância, realçando uma das características mais importantes desta metodologia.

Após encontrar a forma ideal de gerar o cetenimínio, a investigação focou-se na forma de explorar este intermediário, nomeadamente na sua interação com outro tipo de moléculas contendo diversos grupos funcionais. Uma forma de avaliar o potencial do cetenimínio é olhando para a origem da sua palavra. Como o nome indica, cetenimínio é um derivado nitrogenado do ceteno que, por si, tem uma reatividade bastante peculiar, mais especificamente em reações de cicloadição [2+2] (Esquema 14) [41]. No entanto, o seu uso é bastante limitado devido a problemas relacionados com a sua integridade estrutural pois dimeriza com bastante facilidade [42]. 0 mesmo não pode ser dito do intermediário cetenimínio, sendo este, em comparação, muito mais reativo [43].

De forma a elucidar esta situação, Ghosez demonstrou empiricamente que existiam casos em que o cetenimínio reagia de forma excecional, contrastando com a utilização de cetenos. A transformação em questão foi a reação intramolecular [2+2] que forma ciclobutenos após hidrólise do ião imínio [43]. Existem vários fatores que tornam esta reação possível. Se se considerar a estrutura do cetenimínio, observa-se que este parece ter sido especialmente desenhado 
para o efeito, pois a aproximação antarafacial é favorecida devido à geometria (Figura 3).

Outra ilação possível é a de que os grupos adjacentes ao nitrogénio podem permitir a indução de quiralidade na transformação subsequente. Isto acontece devido à proximidade ao centro reativo. Após a cicloadição, estes grupos podem ser facilmente removidos por hidrólise, levando à formação de ciclobutanonas quirais. Esta proposta foi confirmada experimentalmente por Ghosez, tendo o seu grupo testado vários auxiliares quirais obtendo uma transferência de quiralidade satisfatória (Esquema 15) [44]. Os melhores resultados foram obtidos com auxiliares C2 simétricos nas posições 2,5 da pirrolidina. Foi também observado que anéis derivados da pirrolidina são os preferidos para este tipo de reação.

Para além das cicloadições [2+2] mencionadas anteriormente, o cetenimínio pode ser utilizado em conjunto com nucleófilos de carbono para formar diversos compostos interessantes. Do ponto de vista sintético, esta transformação é bastante atraente pois o grupo amida tem uma elevada estabilidade, levando a que seja irresistível a sua utilização numa sequência sintética. Em 2002, o grupo de Overman fez uma interessante descoberta recorrendo ao cetenimínio como intermediário [45]. Ao colocar um alceno numa posição privilegiada, este favoreceu um ataque intramolecular seguido de rearranjo de pinacol, levando à elegante formação de um biciclo 5,7 (Esquema 16). É importante realçar que não foram encontrados vestígios do produto de cicloadição [2+2], muito provavelmente devido ao impedimento estereoquímico.

Outros tipos de nucleófilos de carbono podem ser usados neste tipo de química (particularmente em versão intramolecular), como compostos aromáticos ricos em eletrões, enol éteres [46] e enaminas [47]. De forma complementar, este tipo de transformação também pode ser realizado intermolecularmente, sendo nesse caso mais comum a utilização de reagentes organometálicos, como por exemplo reagentes de Grignard [48]. É ainda possível o uso de diversos nucleófilos,

Figura 3 - Considerações sobre a cicloadição [2+2].

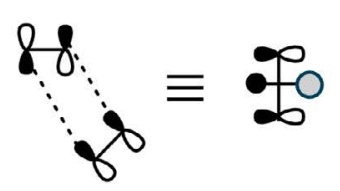

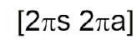

Termicamente permitido

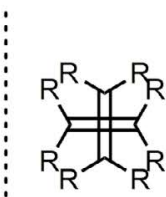

estereamente proibido

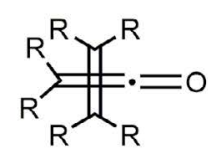

permitido usando ceteno

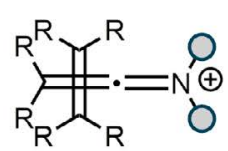

permitido usando cetenimínio

O Possível quiralidade

Esquema 15 - Auxiliares quirais na cicloadição [2+2] [44].<smiles>COC[C@@H]1CCCN1C(=O)O</smiles>

$70 \%$ ee $27 \%$<smiles>CO[C@H]1CN(C(=O)[O-])C[C@H]1OC</smiles>

$75 \%$ rac<smiles>C[C@H]1CC[C@@H](C)N1C(=O)O</smiles>

$62 \%$ ee $92 \%$

Esquema 16 - Rearranjo pinacol alquílico iniciado por ataque de alceno ao cetenimínio (TES = trietilsilil) [45].

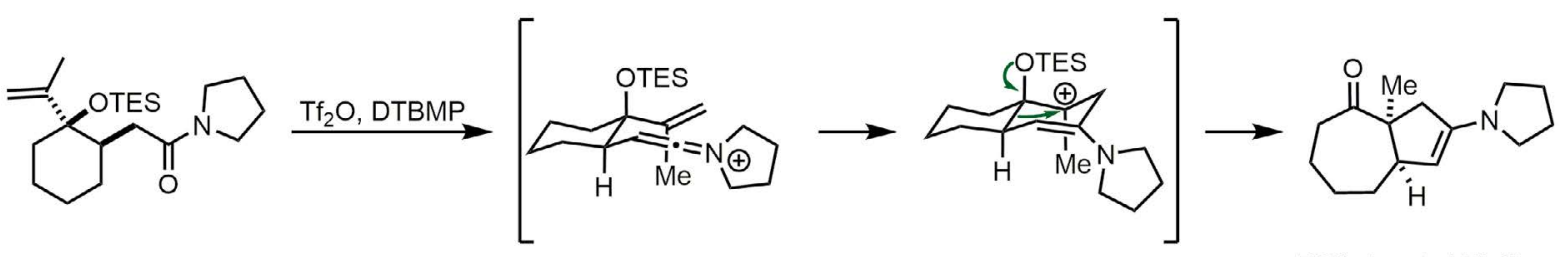


gerando produtos distintos dependendo da ordem de adição dos reagentes, aumentando o potencial desta transformação. Este último foi demonstrado no contexto da síntese total de compostos naturais, nomeadamente (-)-FR901483, onde se verificou uma elevada indução diastereomérica devido ao centro quiral já presente na molécula (Esquema 17) [49].

Outros tipos de reações foram explorados, mais concretamente por Charette, que demonstrou o caráter multifacetado do cetenimínio. Este reagiu com diversos nucleófilos contendo heteroátomos distintos, originando vários grupos funcionais, tais como: ésteres [50], tioamidas [51], amidinas [52], tiazoles [53], triazoles [54] e orto ésteres [55] (Esquema 18). É necessário mencionar que esta metodologia é totalmente quimiosseletiva, levando a que outros grupos funcionais, como ésteres, cetonas e nitrilos, se mantenham inalterados durante a transformação.

Embora partilhe algumas semelhanças, a ativação de amidas secundárias origina diferentes intermediários que podem ser explorados de formas alternativas. Tal como o ião cetenimínio, o ião nitrílio, cuja formação está representada no Esquema 19, é extremamente reativo, sendo facilmente capturado por diversos nucleófilos. Uma das grandes famílias de compostos a que a ativação de amidas secundárias dá acesso é a dos heterociclos, onde é possível incorporar o átomo de nitrogénio da amida no esqueleto da molécula-alvo.<smiles>C=CCN1C[C@H](OCc2ccccc2)[C@H](OCCC)CCC1=O</smiles>

(-)-FR901483

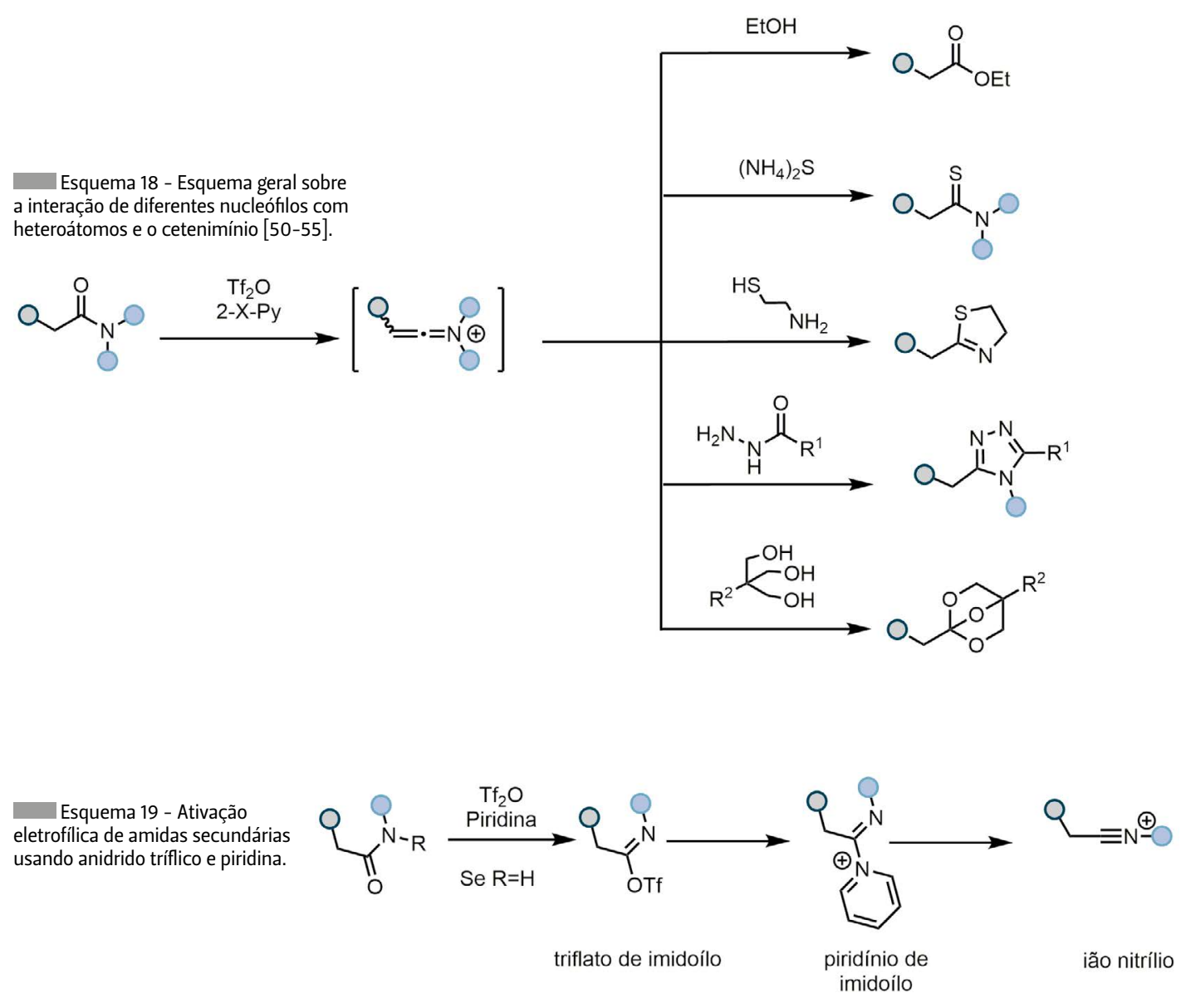




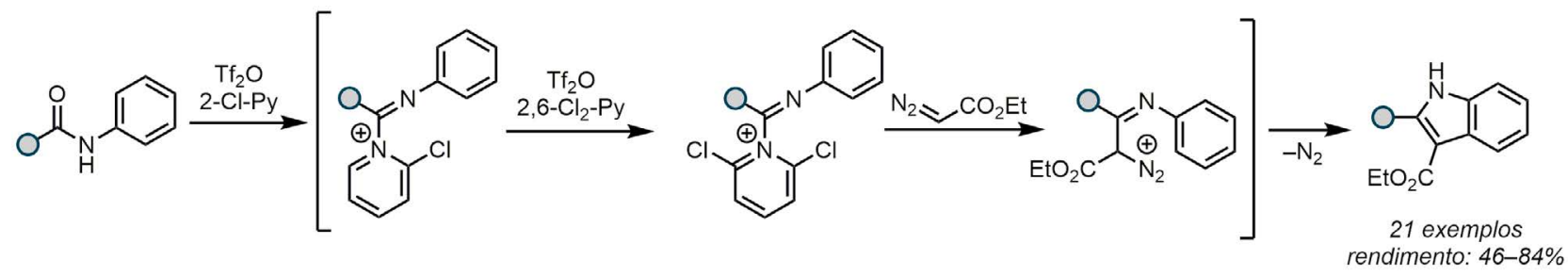

a

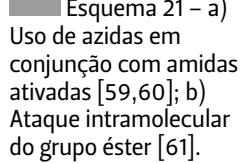

do grupo éster [61].

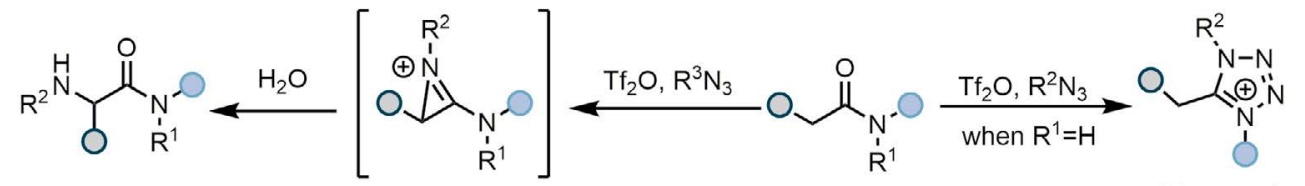

21 exemplos rendimento: $41-99 \%$

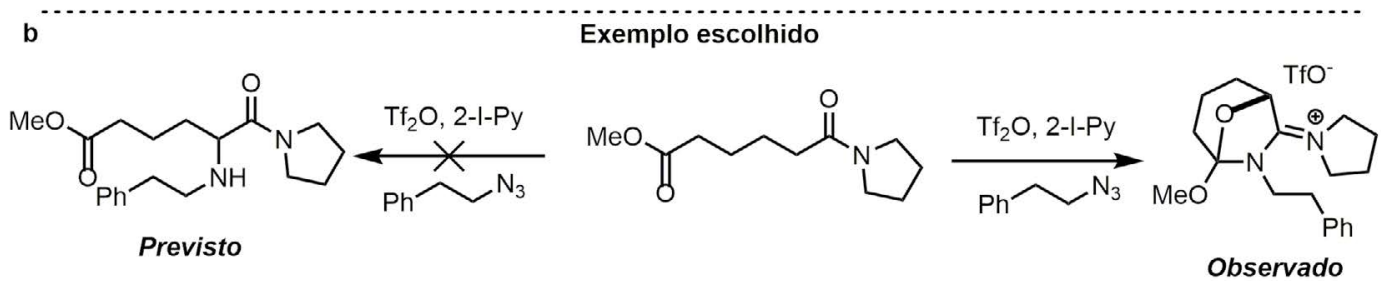

rendimento: $31 \%$

A síntese de compostos heterocíclicos tem sido ao longo dos anos uma área que suscita interesse, muito devido ao seu impacto na indústria farmacêutica. Este tipo de estrutura confere, a uma grande maioria dos fármacos, certas propriedades que os tornam únicos em termos de atividade e estabilidade. Alguns dos exemplos mais proeminentes são os indoles [56], as pirimidinas [57] e as piridinas [58]. Curiosamente, estes importantes alvos sintéticos conseguem ser facilmente sintetizados com a ativação de amidas secundárias. Um interessante caso de estudo é a síntese de indoles [56] (Esquema 20) em que a otimização de todos os componentes da reação é crucial para que o rendimento seja satisfatório.

Tal como mencionado anteriormente, a escolha apropriada da base halogenada é especialmente importante para este tipo de transformações. Neste caso em particular, uma combinação de duas bases é usada com elevado sucesso. A ativação inicial necessita de 2-cloropiridina para ocorrer. No entanto, os autores observaram que o diazo-éster não consegue atacar o intermediário, levando a uma baixa conversão [56]. A solução foi a adição de uma base mais eletrodeficiente que favorece o ataque anteriormente mencionado, solucionando o problema.

No contexto da dualidade entre amidas terciárias e secundárias existe uma reação que elucida comple- tamente a diferença entre as espécies que, à partida, parecem ser tão semelhantes. Quando o intermediário nitrílio, derivado da amida secundária, reage com azidas, uma reação de cicloadição é comummente observada, levando à formação de tetrazoles. Este facto foi descoberto em 1993 com o ácido hidrazóico [59] mas só foi plenamente explorado em 2007 por Maulide. Nesse caso em específico, recorreu-se a azidas alquílicas para formar os sais correspondentes, sais de tetrazónio [60].

Comparativamente, na reatividade do ião cetenimínio com azidas uma reação completamente distinta é observada, levando à formação de aminas, formalmente uma $\alpha$-funcionalização da amida [61]. Na presença de água, a azirina fraǵmenta levando à formação de uma amina secundária com libertação de $\mathrm{N}_{2}$ (Esquema 21a). Uma importante descoberta foi feita quando os autores reportaram que este intermediário podia ser intercetado por um grupo éster distal, levando à formação de um composto bicíclico (Esquema 21b). Este facto abriu os horizontes para a exploração deste tipo de química onde um composto antagónico ao enolato, enolónio, é formado.

Uma forma direta de chegar a um intermediário bastante semelhante baseia-se na utilização de N-óxidos, cuja interação com o cetenimínio resulta numa espécie denominada de enolónio. Este intermediário pode ainda 
ser obtido através da adição em excesso do N-óxido [61], levando à formação de $\alpha$-piridínios, que podem ser clivados gerando o álcool, ou oxidados à cetona correspondente. Um vasto número de nucleófilos foi estudado em combinação com o enolónio (Esquema 22) [62-65]. Um dos exemplos iniciais foi com arenos eletronicamente ricos [62] que, curiosamente, necessitaram de temperaturas bastante elevadas para a reação ser completa. Posteriormente foi descoberto que, devido à inerente eletrodeficiência do enolónio, este é capturado rapidamente pelo contra-ião triflato, que advém do anidrido tríflico usado na ativação da amida [64]. Isto explica o porquê da necessidade de temperaturas elevadas para o ataque de compostos aromáticos ricos em eletrões, que necessita ser facilitada adicionalmente por uma base.

Relativamente à formação da ligação carbono-carbono, também foi observado que este método era compatível com diversos enolatos, formando diretamente 1,4-dicarbonilos [65]. $0 \alpha$-triflato gerado pode ser explorado de diversas maneiras, especialmente quando combinado com diferentes nucleófilos. Um dos primeiros exemplos da manipulação deste triflato lábil foi no acoplamento do tipo Suzuki, onde estes triflatos são, em primeiro lugar, transformados em brometos devido à sua baixa estabilidade e, depois, combinados com níquel e ácidos borónicos [66]. Outros halogéneos podem ser usados nesta reação, resultando, na maioria dos casos, em rendimentos excelentes. Um caso específico que gerou bastante entusiasmo foi a utilização de fluoreto (Esquema 23). A possibilidade de fluorar nucleofilicamente amidas pode ser crucial para a descoberta de novos mecanismos de ação de fármacos no organismo, devido à chamada Hot Fluorination [67]. Este método faz uso do isótopo ${ }^{18} \mathrm{~F}$ para monitorizar por scan de PET (tomografia eletrónica de positrão) as áreas de acumulação do composto fluorado, permitindo aplicações em técnicas de diagnóstico e imagem médicas. Por fim, este método pode ainda ser aplicado na síntese de derivados fluorados de moléculas importantes, tais como citalopram, piperalina e fentanilo.

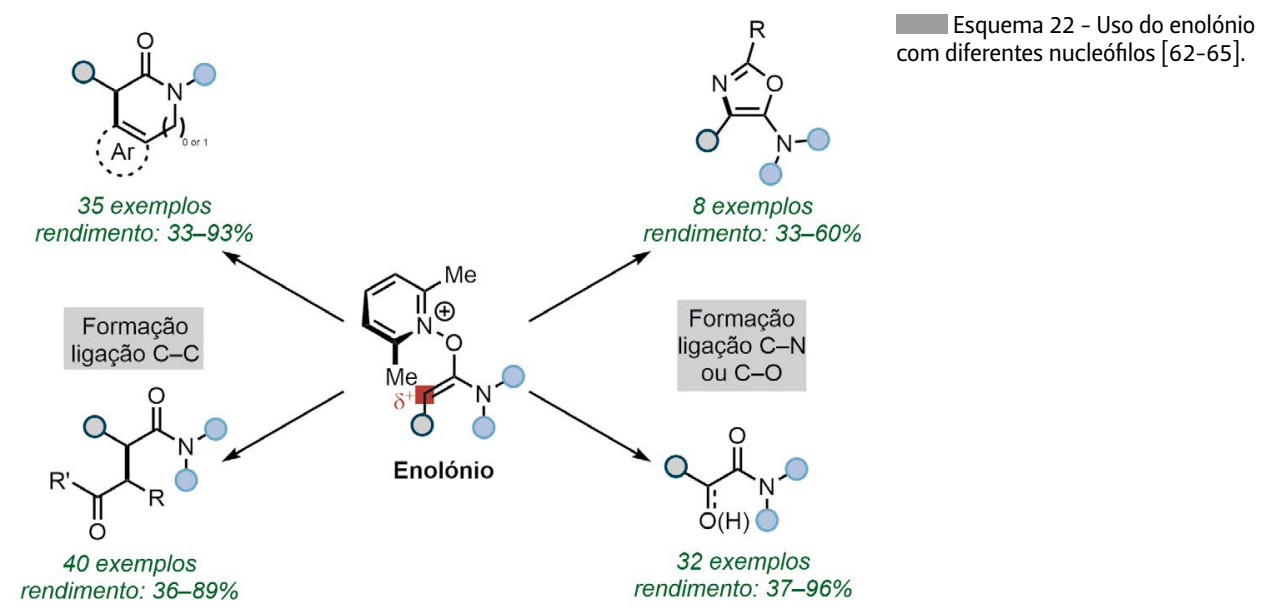

a
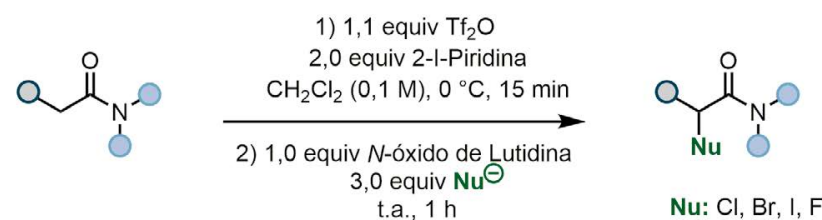

Esquema 23 - a) Funcionalização do enolónio usando diferentes halogéneos. b Moléculas relevantes obtidas após fluoração [67].

$\mathrm{Nu}: \mathrm{Cl}, \mathrm{Br}, \mathrm{I}, \mathrm{F}$

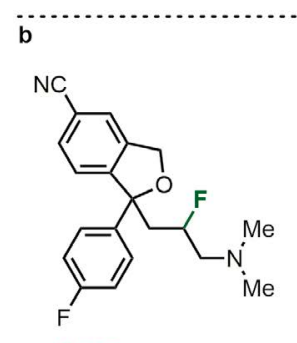

fluoro-citalopram

Antidepressivo

Exemplos Fluorados

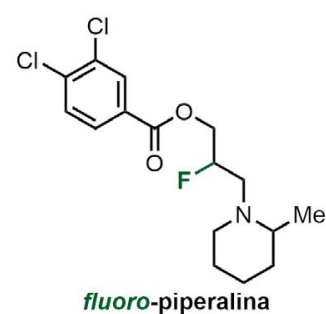

Agroquímico

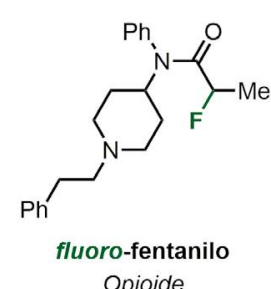

Opioide 


\section{Conclusões}

Com esta revisão, pretendeu-se mostrar a ponta do iceberg que é a reatividade versátil do grupo funcional amida. A forma equívoca de considerar a ligação amida como algo imutável leva a que a sua manipulação seja ainda mais valiosa pois, ultrapassados estes paradigmas, novas soluções podem ser encontradas.

\section{Agradecimentos}

Os autores agradecem ao Professor Nuno Maulide e ao Dr. Alexandre Pinto pela ajuda na preparação do manuscrito. Os autores agradecem ainda à Professora Diana Gonçalves, Hugo Lisboa e à Dora pelo seu incansável apoio na revisão deste artigo.

\section{Referências}

[1] M. Rueping, B. J. Nachtsheim, Beilstein J. Org. Chem. 2010, 6, 1-24. DOI: 10.3762/bjoc.6.6.

[2] a) C. Friedel, J. -M. Crafts, Compt. Rend. 1877, 84, 1450-1454. biodiversitylibrary.org/item/23739\#page/1458/mode/1up; b) C. Friedel, J. -M. Crafts, Compt. Rend. 1877, 85, 673-676. biodiversitylibrary.org/ item/24548\#page/685/mode/1up.

[3] C. Friedel, J. -M. Crafts, Compt. Rend. 1877, 85, 74-77. biodiversitylibrary. org/item/24548\#page/80/mode/1up.

[4] S. Prajapati, A. P. Mishra, A. Srivastava, Int. J. Pharm. Chem. Biol. Sci. 2012, 2 , 52-62. /ijpcbs.com/files/volume2-1-2012/05.pdf.

[5] N. Weires, E. Baker, N. Garg, Nat. Chem. 2016, 8, 75-79. DOI: 10.1038/ nchem.2388

[6] M. M. Mehta, T. B. Boit, J. E. Dander, N. K. Garg, Org. Lett. 2020, 22, 1-5. DOI: 10.1021/acs.orglett.9b03434.

[7] T. B. Boit, N. A. Weires, J. Kim, N. K. Garg, ACS Catal. 2018, 8, 1003-1008. DOl:10.1021/acscatal.7b03688.

[8] C. Liu, M. Szostak, Chem. Eur. J. 2017, 23, 7157-7173. DOI: 10.1002 chem.201605012

[9] A. J. Kirby, I. V. Komarov, P. D. Wothers, N. Feeder, Angew. Chem., Int. Ed., 1998, 37, 785-786.. DOI: 10.1002/(SICI)1521-3773(19980403) 37:6<785::AID-ANIE785>3.0.CO;2-J.

[10] K. Tani, B. Stoltz, Nature 2006, 441, 731-734., DOI: 10.1038/nature04842.

[11] R. Lukeš, Collect. Czech. Chem. Commun. 1938, 10, 148-152. D0I: 10.1135/ cccc19380148.

[12] H. H. Wasserman, Nature 2006, 441, 699-700. DOI: 10.1038/441699a.

[13] Y. Lei, A. D. Wrobleski, J. E. Golden, D. R. Powell, J. Aubé, J. Am. Chem. Soc. 2005, 127, 4552-4553. DOI: 10.1021/ja050214m.

[14] G. Meng, P. Lei, M. Szostak, Org. Lett. 2017, 19, 2158-2161. DOI: 10.1021/acs. orglett.7b00796

[15] G. Li, M. Szostak, Synthesis 2020, 52, 2579-2599. DOI: 10.1055/s-0040-1707101.

[16] V. Pace, W. Holzer, G. Meng, S. Shi, R. Lalancette, R. Szostak, M. Szostak, Chem. Eur. J. 2016, 22, 14494-14498. DOI: 10.1002/chem.201603543.

[17] a) A. Greenberg, C. Breneman, M. J. F. Liebman, "The Amide Linkage: Structural Significance in Chemistry, Biochemistry, and Materials Science", Wiley, New York, 2000; b) S. D. Roughley, A. M. J. Jordan, Med. Chem. 2011 54, 3451-3379. DOI: 10.1021/jm200187y; c) K. Marchildon, Macromol. React. Eng. 2011, 5, 22-54. DOI: 10.1002/mren.201000017; d) V. R. Pattabiraman, J. W. Bode, Nature 2011, 480, 471-479. DOI: 10.1038/ nature10702; e) B. Trost, M. Fleming, "Comprehensive Organic Synthesis", Pergamon Press, Oxford, 1991.

[18] J. Dander, E. Baker, N. Garg, Chem. Sci. 2017, 8, 6433-6438. DOI: 10.1039/ C7SC01980G.

[19] J. Dander, N. Garg, ACS Catal. 2017, 7, 1413-1423. DOI: 10.1021/acscatal. 6b03277.

[20] Á. L. F. de Arriba, E. Lenci, M. Sonawane, O. Formery, D. J. Dixon, Angew. Chem. Int. Ed. 2017, 56, 3655-3659. DOI: 10.1002/anie.201612367.
[21] D. M.-Raven, P. Gabriel, J. A. Leitch, Y. A. Almehmadi, K. Yamazaki, D. J. Dixon, ACS Catal. 2020, 10, 8880-8897. DOl: 10.1021/acscatal.0c02377.

[22] B. J. Simmons, M. Hoffmann, J. Hwang, M. K. Jackl, N. K. Garg, Org. Lett. 2017, 19, 1910-1913. D0I: 10.1021/acs.orglett.7b00683.

[23] T. B. Boit, M. M. Mehta, J. Kim, E. L. Baker, N. K. Garg, Angew. Chem. Int. Ed. 2021, 60, 2472-2477. DOI: 10.1002/anie.202012048.

[24] J. E. Dander, M. Giroud, S. Racine, Commun. Chem. 2019, 82, 1-9. DOI: 10.1038/s42004-019-0182-8.

[25] S. Shi, G. Meng, M. Szostak, Angew. Chem. Int. Ed. 2016, 55, 6959-6963. DOI: 10.1002/anie.201601914.

[26] S. Shi, M. Szostak, Org. Lett. 2017, 19, 3095-3098. DOI: 10.1021/acs. orglett.7b01199.

[27] C. Liu, M. Szostak, Angew. Chem. Int. Ed. 2017, 56, 12718-12722. DOI: 10.1002/anie.201707102

[28] G. Meng, M. Szostak, Org. Lett. 2015, 17, 4364-4367. DOI: 10.1021/acs orglett.5b02209.

[29] G. Li, M. Szostak, Chem. Rec. 2020, 20, 649-659. D0I: 10.1002/ tcr.201900072

[30] Y. Liu, S. Shi, M. Achtenhagen, R. Liu, M. Szostak, Org. Lett. 2017, 19, 1614-1617. DOI: 10.1021/acs.orglett.7b00429.

[31] G. Li, M. Szostak, Nat. Commun. 2018, 9, 4165. D0I: 10.1038/s41467-01806623-1.

[32] G. Li, P. Lei, M. Szostak, Org. Lett. 2018, 20, 5622-5625. DOI: 10.1021/acs. orglett.8b02323.

[33] D. Y. Ong. D. Fan, D. J. Dixon, S. Chiba, Angew. Chem. Int. Ed. 2020, 59 11903-11907. DOl: 10.1002/anie.202004272.

[34] A. Bischler, B. Napieralski, Ber. Dtsch. Chem. Ges. 1893, 26, 1903-1908. DOI: 10.1002/cber.189302602143.

[35] T. Figee, Recl. Trav. Chim. Pays-Bas 1915, 34, 289-325. D0l: 10.1002/ recl.19150341002

[36] L. Ghosez, B. Haveaux, H. G. Viehe, Angew. Chem. Int. Ed. 1969, 8, 454-455. DOI: 10.1002/anie.196904541.

[37] J.-B. Falmagne, J. Escudero, S. T.-Sahraoui, L. Ghosez, Angew. Chem. Int. Ed. Engl. 1981, 20, 879-880. DOI: 10.1002/anie.198108791.

[38] a) B. Peng, D. Geerdink, C. Farès, N. Maulide, Angew. Chem. Int. Ed. 2014 53, 5462-5466. DOl: 10.1002/anie.201402229; b) K. L. White, M. Mewald, M. Movassaghi, J. Org. Chem. 2015, 80, 7403-7411. DOI: 10.1021/acs. joc.5b01023.

[39] A. B. Charette, M. Grenon, Can. J. Chem. 2001, 79, 1694-1703. D0l: 10.1139/v01-150.

[40] H. Staudinger, "Die Ketene", Verlag von Ferdinand Enke, Stuttgart, 1912.

[41] L. Tenud, M. Weilenmann, E. Dallwigk, Helv. Chim. Acta 1977, 60, 975-977. DOI:10.1002/hlca.19770600328.

[42] H. K. Hall, C. D. Smith, D. E. Plorde, J. Org. Chem. 1973, 38, 2084-2087. DOl: 10.1021/j000951a024 
[43] a) J. M.-Brynaert, L. Ghosez, J. Am. Chem. Soc. 1972, 94, 2870-2872. D0 10.1021/ja00763a062; b) J. Falmagne, J. Escudero, S. T.-Sahraoui, L. Ghosez, Angew. Chem. Int. Ed. 1981, 20, 879-880. DOI: 10.1002/ anie.198108791.

[44] L. Chen, L. Ghosez, Tetrahedron Lett. 1990, 31, 4467-4470. DOI: 10.1016/ So040-4039(00)97650-0

[45] L. E. Overman, J. P. Wolfe, J. Org. Chem. 2002, 67, 6421-6429. DOI: 10.1021/ jo025927r.

[46] K. J. Xiao, J. M. Luo, K. Y. Ye, Y. Wang, P. Huang, Angew. Chem. Int. Ed. 2010, 49, 3037-3040. DOI: 10.1002/anie.201000652.

[47] F. Barabé, M. Nantel, R. L.-Gauthier, G. Bélanger, Org. Lett. 2005, 7, 4431-4434, DOI: 10.1021/ol0516519.

[48] K. J. Xiao, J. M. Luo, K. Y. Ye, Y. Wang, P. W. Huang, Angew. Chem. Int. Ed. 2010, 49, 3037-3040. DOI: 10.1002/anie.201000652

[49] H. H. Huo, X. E. Xia, H. K. Zhang, P. Q. Huang, J. Org. Chem. 2013, 78 455-465. DOI: 10.1021/j0302362b.

[50] A. B. Charette, P. Chua, Synlett 1998, 2, 163-165. DOI: 10.1055/s-1998-1612.

[51] A. Charette, P. Chua, Tetrahedron Lett. 1998, 39, 245-248. D0I: 10.1016/ S0040-4039(97)10505-6.

[52] A. B. Charette, M. Grenon, Tetrahedron Lett. 2000, 41, 1677-1680. DOI: 10.1016/S0040-4039(00)00040-X

[53] A. B. Charette, P. Chua, J. Org. Chem. 1998, 63, 908-909. DOI: 10.1021/ j09718830.

[54] W. S. Bechara, I. S. Khazhieva, E. Rodriguez, A. B. Charette, Org. Lett. 2015, 17, 1184-1187. DOI: 10.1021/acs.orglett.5b00128.

[55] A. Charette, P. Chua, Tetrahedron Lett. 1997, 38, 8499-8502. DOI: 10.1016/ S0040-4039(97)10310-0.
[56] S. L. Cui, J. Wang, Y. G. Wang, J. Am. Chem. Soc. 2008, 130, 13526-13527. DOI: 10.1021/ja805706r.

[57] M. D. Hill, M. Movassaghi, J. Am. Chem. Soc. 2006, 128, 14254-14255. DOI: $10.1021 / \mathrm{ja} 066405 \mathrm{~m}$.

[58] O. K. Ahmad, M. D. Hill, M. Movassaghi, J. Am. Chem. Soc. 2007, 129, 1009610097. DOI: 10.1021/ja073912a.

[59] E. W. Thomas, Synthesis 1993, 8, 767-768. DOI: 10.1055/s-1993-25934.

[60] V. Tona, B. Maryasin, A. D. L. Torre, J. Sprachmann, L. González, N. Maulide, Org. Lett. 2017, 19, 2662-2665. DOI: 10.1021/acs.orglett.7b01004.

[61] V. Tona, A. D. L. Torre, M. Padmanaban, S. Ruider, L. González, N. Maulide, J. Am. Chem. Soc. 2016, 138, 8348-8351. DOI: 10.1021/jacs.6b04061.

[62] A. D. L. Torre, D. Kaiser, N. Maulide, J. Am. Chem. Soc. 2017, 139, 6578-6581. DOI: 10.1021/jacs.7b02983.

[63] D. Kaiser, A. D. L. Torre, S. Shaaban, N. Maulide, Angew. Chem. Int. Ed. 2017, 56, 5921-5925. DOI: 10.1002/anie.201701538.

[64] C. R. Gonçalves, M. Lemmerer, C. J. Teskey, P. Adler, D. Kaiser, B. Maryasin, L. González, N. Maulide, J. Am. Chem. Soc. 2019, 141, 18437-18443. DOI: 10.1021/jacs.9b06956.

[65] D. Kaiser, C. J. Teskey, P. Adler, N. Maulide, J. Am. Chem. Soc. 2017, 139 16040-16043. DOI: 10.1021/jacs.7b08813.

[66] J. Li, M. Berger, W. Zawodny, M. Simaan, N. Maulide, Chem. 2019, 5, 18831891. DOI: 10.1016/j.chempr.2019.05.006.

[67] P. Adler, C. J. Teskey, D. Kaiser, M. Holy, H. H. Sitte, N. Maulide, Nat. Chem. 2019, 11, 329-334. DOI: 10.1038/s41557-019-0215-z.

\section{Ana Mortinho}

Faculdade de Ciências e Tecnologia, NOVA School of Science \& Technology Ana Mortinho é Mestre em Química Bioorgânica pela Faculdade de Ciências e Tecnologia da Universidade NOVA de Lisboa. Desenvolveu a sua tese de mestrado com a Professora Maria Manuel Marques sobre a síntese de heterociclos recorrendo a catálise metálica. Trabalhou recentemente com o Professor Paul Knochel na aplicação de compostos organometálicos para a síntese de arenos e heteroarenos substituídos. a.mortinho@campus.fct.unl.pt ORCID.org/0000-0002-1514-0159

\section{* Carlos R. Gonçalves}

Instituto de Química Orgânica, Universidade de Viena.

Carlos Rafael Gonçalves é Mestre em Química Bioorgânica pela Faculdade de Ciências e Tecnologia da Universidade NOVA de Lisboa. A sua tese de mestrado baseou-se na síntese de sondas fluorescentes e em novas metodologias de aminação, com a Professora Maria Manuel Marques e o Professor Nuno Maulide. Atualmente é estudante de doutoramento na Universidade de Viena sob orientação do Professor Nuno Maulide. carlos.goncalves@univie.ac.at ORCID.org/0000-0003-1719-1969. 